\title{
Towards a Smart Port: The Role of the Telecom Industry
}

\author{
Christos-Antonios Gizelis ${ }^{1(\bowtie)}(\mathbb{D})$, Theodoros Mavroeidakos $^{1}(\mathrm{D}$, \\ Achilleas Marinakis ${ }^{2}$ (D), Antonis Litke ${ }^{2}$ (D), and Vrettos Moulos ${ }^{2}$ (D) \\ 1 Hellenic Telecommunications Organization S.A., \\ 99 Kifissias Avenue, Athens, Greece \\ cgkizelis@cosmote.gr, tmavroeid@ote.gr \\ 2 National (Metsovian) Technical University of Athens, \\ Patision Complex, Athens, Greece \\ \{achmarin, litke, vrettos\}@mail.ntua.gr \\ http://www.ote.gr, http://www.ece.ntua.gr
}

\begin{abstract}
Transformation is not only today's trend but also a reality. Ports could not be excluded from that change. A transformation process has been initiated in order to change their operational structure, and the services they offer. Artificial Intelligent and Data oriented services push the services's landscape beyond the traditional ones that are currently used. The scope of this paper is to analyze and scrutinize the opportunities that are risen for Telecommunications/Information and Communication Technology (ICT) providers at ports. These opportunities are the stepping stone towards the transformation of ports for the future. This work in progress is under the DataPorts project that is funded by the European Union's Horizon 2020 Research and Innovation Programme under grant agreement No. 871493.
\end{abstract}

Keywords: Ports $\cdot$ Smart Ports - Data platforms · AI - Blockchain • Privacy

\section{Introduction}

"DataPorts project aims to boost the transition of European seaports from connected and digital to smart and cognitive, by providing a secure environment for the aggregation and integration of data coming from different sources existing in the digital ports and owned by diverse stakeholders, so that the whole port community could benefit from this data in order to improve their processes, offer new services and devise new AI based and data driven business models" [10]. For this purpose, the technological innovation is destined to transform the business as usual, therefore more and more companies hop on that huge wave in order to avoid to be forgotten in the near future as it happened in well branded companies in the past that did not see the need for change $[16,20]$. This massive transformation of the businesses has become more data-driven. Inevitable data owners 
or those that can produce data have become the new major actors. The port industry is no exception and data-driven services is what they should offer, not in the future but today to their end-user, customers, stakeholder (many names exist to define them). According to Deloitte Port Services [3], Smart Ports is the fourth technological priority in improving and evolving the Shipping/Maritime Industry. By making a port smart, then AI-based services will offer cognitive services that will offer opportunities to Port owners, visitors, customers, etc.

Smart and Cognitive Ports is the newest trend and like the Smart Cities is a creation of a new emerging data market. It is a term that expands the traditional stakeholders' ecosystem with limitless opportunities for new entries. A multiactor and very diverse ecosystem is created with many opportunities for market expansion, revenue increase, new services, especially data-driven ones such as Internet of Things (IoT) [30] and AI-based services. This rapid growing ecosystem with many actors and many roles creates the need for new and dynamic Business Models to fulfill the also rising needs. This opportunity was early identified by European Union in 2014 [29] and a special chapter was included within the Smart Cities one. "Ports are considered a special case of a Smart Community, then they have to meet the same requirements that are asked for a Smart City, adapted to the port situation".

Ports operate on a certain basis by following a number of models. They can be Public, where the administration is operated by a central authority at a national, regional or city level, or Private, a model that is run by a port company, or even a port (local) society and Hybrid where in this case, public-private partnerships govern the port administration and the provided services. Therefore, Port Authorities whether are Private, Public, or Hybrid, are "forced" to transform and create the Ports of the future, not only by creating an interconnection grid between them but offering new innovative services to their existing "customers" like companies in shipping, supply-chain and logistics, tourism, but also many more from a wide diversity areas, that they can take advantage from the new services. Therefore, Port Authorities create synergies with research community, data owners and providers, software-houses, startups and SMEs in order to, together create new innovative services and expand the list of the potential beneficiaries.

The need of Port Authorities has become an opportunity for port-oriented companies to experiment their wide range of services, algorithms, and data that they own and monetize their offerings in this new emerging market. It is an opportunity, which could be beneficial for numerous stakeholders. This opportunity increases the dynamics of the Smart Ports or Ports of the Future transformation.

The basis for port transformation lays within the co-operation that takes place between strategic partnerships at national and international level. To that we should add partnerships with port-oriented service 3 rd parties. The new ecosystem that is created around the ports is highly competitive in order to increase their market share, and ports are trying to get an advantage by implementing smart technologies and services in order to optimize their operations in 
many areas such as energy, security, transport, logistics and more. Local Port Authorities gather around, numerous entities. Organizations, Associations, Companies that may be stakeholders and potentially beneficiaries of a data-oriented platform provided by DataPorts. The list can be endless if attempt to put everyone in the frame, but trying to pin-point the main ones we can identify synergies within Academia and Research Community, Shipping companies, SMEs and Startups, Public Authorities and Policy Makers, Data Providers, and Local Community Associations (Commercial, Tourism, Culture, etc.)

On the port transformation journey, the Telecommunication industry (also involved in ICT services) can have a significant role and perhaps become a key player towards the transformation success. Telecom (wired and wireless) hold the backbone of the data-driven environment $[1,2,7]$ through their networks. Therefore, it is on their hand to become leaders in this rapidly increased emerging market. A Telecom/ICT Provider in order to enter this emerging ecosystem and potentially benefit from its growth should firstly address real-life data market use cases in Ports that are related to its areas of operations. The data that a Telecom Provider owns might not be directly usable by Ports' community and interoperability issues should be prior investigated. Data may be provided/shared through advanced AI-based services that are scalable, resilient and using a semantic approach. Data should be designed and provided in a matter that can be governed and maintained in a business manner. For these reasons, new business models and a standardized methodology like [21] should be defined and adopted respectively. Policies should be also taken under consideration so that a reliable data sharing and trading platform to be established. These design patterns should be obtained under a secure and trusted environment between stakeholder and beneficiaries, internal or external to the Port. Telecommunication industry owns, due to its field of operations, owns, handles and process large volumes of data, real and non-real time. Data that collected and stored from many different services. A Telecom Provider has the largest availability of customers' personal data, network data, mobility tracking, billing location, and so on. The correlation of such datasets, as well as the combination of them with external publicly available data, may potentially create a great value for solving problems and serving future needs. Since it is a new field of operations and due to the fact that until now such data sharing wasn't available for many different reasons, the value or what needs data can solve may be uncertain yet. According to the EU [29], it is estimated that the open data market is expected to increase by $37 \%$ by 2020 , reaching $€ 75.7$ billion, with the cumulative figure of $€ 325 \mathrm{bn}$. Open data in Greece can generate an additional $€ 3.2 \mathrm{bn}$. GDP and approximately $€ 12$ billion in cumulative benefits within 5 years. That led researchers, and other related entities to be highly involved, in order benefit from these numbers.

\section{Related Work}

Every Port transformation was focused on different elements. From the Freight loading and uploading in the 1960s, the industrial port and the logistics in the 
1980s. Today even with a tighter regulatory environment from the data sharing point of view, the element of transformation is the interoperability and how the ports of the future will become smart and cognitive. The research community and the port related actors are relative active during the last few years. European Authorities turned their attention into three points of action that could potentially affect and transform a Port; the supply, the Platform and the Demand. Various Port Management Platforms/Services/Applications/Systems, where created in order to cover these elements. Supply in order to monitor and handle the operations with the logistics companies, the shipping companies and the freight carriers. Port operations are monitored both for inbound and outbound activities, both equally demanding with different actors in each value chain. Several initiatives in European level, have taken place in the past and some of them are very active even today in order to create an ecosystem around the Ports. Actions of European Organizations and Associations such as ESPO [13], IAPH [15] and AIVP [28] aim to interconnect and represent Port Authorities and their relationships with EU and other States. They played a vital role in the global trade and practically can be considered as the pioneers of the Smart Ports. In addition to that, ENISA [11] developed - in collaboration with several EU ports - a report that intends to provide a useful information regarding the cybersecurity strategy of port authorities and terminal operators.

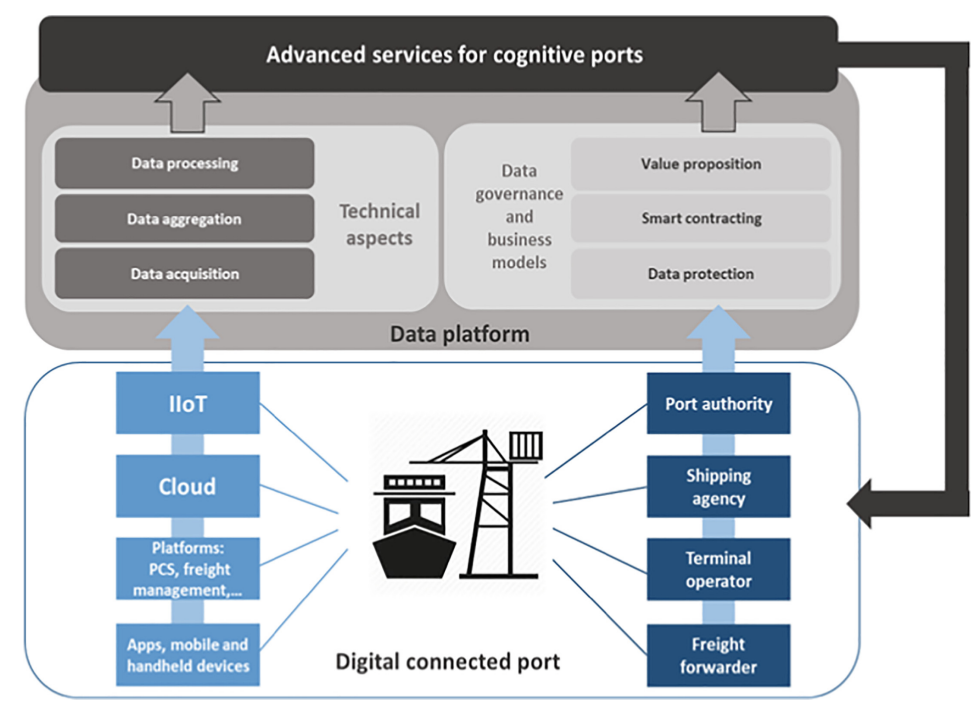

Fig. 1. DataPorts concept

Moreover, EU - especially through Horizon2020 programs - has funded numerous projects, that aim to create management platforms [17] for maritime and port environments, in order to create interoperability and eventually the 
ports to become cognitive and smart. Projects like SmartCities, among others, became The Marketplace of the European Innovation Partnership on Smart Cities and Communities [27]. The projects e-Mar, FLAGSHIP, and INMARE [9] handle maritime transport related issues, MASS [9] deals with ways to improve human behavior on board ships with special attention to emergency situations, MARINE-ABC [9] demonstrates the opportunities of the mobile ship-to-shore communication, BigDataStack [5] attempts to optimize cluster management for data operations, SmartShip [25] develops data analytics decision support systems and a circular economy based optimization platform. All the aforementioned and other projects prove that research community, port authorities, shipping and supply companies are aligned with a common objective which is the creation of a new ecosystem with advanced data-driven services for the benefit of the ports and the local communities. On top of that, European maritime sector through new calls is planning to offer efficient quality services integrated to the overall European transport system.

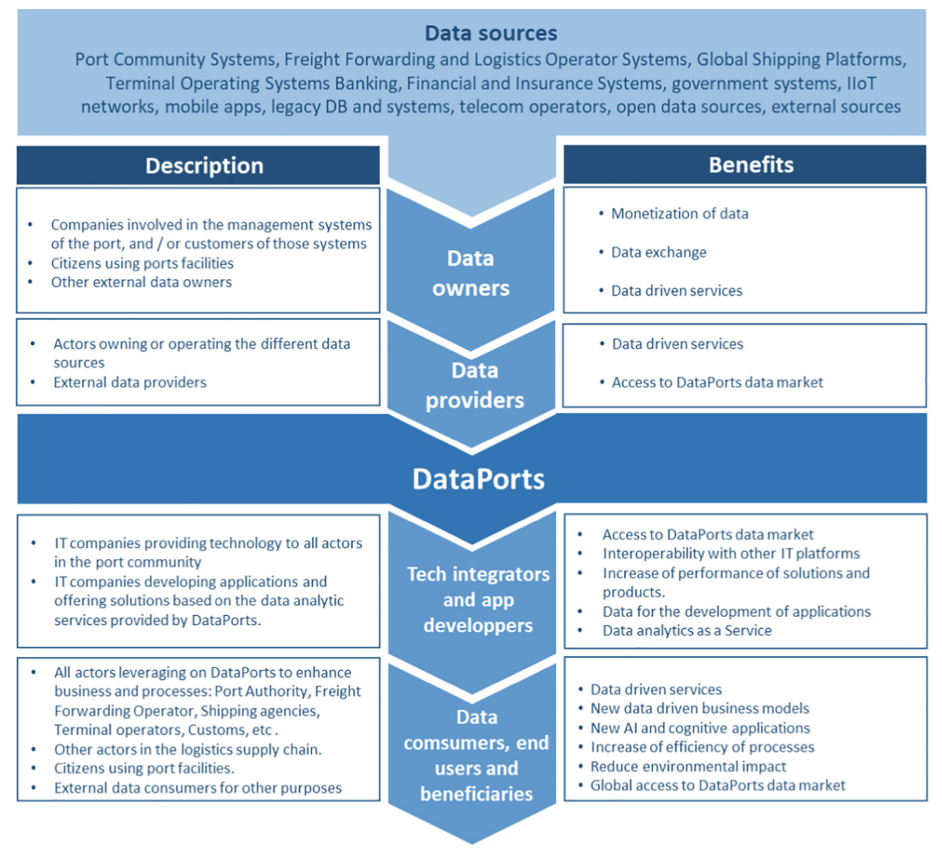

Fig. 2. DataPorts value-chain and stakeholders

DataPorts since January 2020 is planning to implement a data management platform to be operated by Port Authorities in order to provide advanced services (Fig. 1) and create a value-chain between stakeholders, internal and external ones (Fig. 2). 


\section{DataPorts Conceptualization}

Data in the maritime domain is growing at an unprecedented rate, e.g., terabytes of oceanographic data are collected every month as well as petabytes of data are already publicly available. Big data from different sources such as sensors, buoys, vessels, and satellites could potentially feed a large number of interesting applications regarding environmental protection, security, shipping routes optimization or cargo handling [18]. Although many projects [24,25] are trying to develop data management platforms in various application domains, not many of them have addressed integration in port environments with the possibility of including cognitive services and extending their platform to whole transportation routes around Europe.

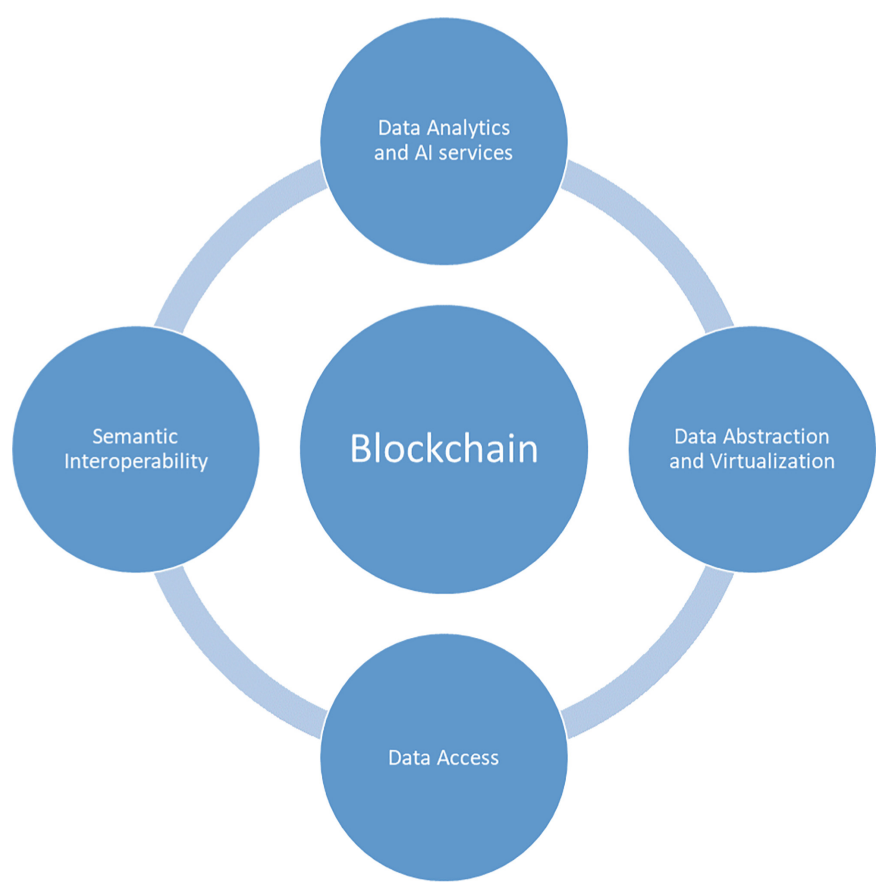

Fig. 3. DataPorts architecture

To this end, Fig. 3 presents the main building blocks of the DataPorts platform's proposed solution:

- Data Access: the platform will ensure access to the heterogeneous data sources of the port - including relational and NoSQL databases, object storage, publish/subscribe streams - in a common manner. To achieve this goal, the project will rely on widely adopted data formats and interface specifications, such as JSON [26] and OpenAPI [22] respectively. 
- Semantic Interoperability: the project will develop a framework for semantic interoperability of diverse data platforms, autonomous systems and applications. Acting as a unifier, the framework will provide business level interoperability, in addition to data interoperability at the level of common spatial and temporal semantics.

- Data Abstraction and Virtualization: the platform will provide an abstraction layer that takes care of retrieving, processing and delivering the data with the proper quality level, while in parallel putting special emphasis on data security, performance, privacy and protection. This layer, acting as a middleware, will let the data consumers simply define their requirements on the needed data - expressed as data utility - and will take the responsibility for providing these data timely, securely and accurately by hiding the complexity of the underlying infrastructure. The latter could consist of different platforms, storage systems, and network capabilities. The orchestration of those (micro)services could be implemented via flow-based programming tools like Node-RED [23].

- Data Analytics and AI services: the project will develop a set of data analytics services for supporting the development of descriptive, predictive, prescriptive models using the different datasets available to the platform. Since the project will have to deal with big data technologies, the defined services should be scalable enough to process huge data volumes. Appropriate State-of-the-Art machine learning algorithms will be identified for supporting the development of cognitive applications in the context of the smart ports domain.

- Blockchain: the platform will provide all the tools for data sharing and trading in a secure and trusted manner. The specific building block should take into consideration the rules defined from data providers to data consumers. On top of that, it has to offer a clear value proposition to data owners. Data access mechanisms, based on purpose control, will also be established. As a result, the solution has to keep provenance of the data entering the platform and implement the functionalities of data governance. The project will exploit permissioned blockchain technology such as Hyperledger [14], in order to address all these requirements.

\section{Challenges and Opportunities}

As mentioned earlier in this paper, the emerging data market within ports is very appealing for a telecommunications provider due to the wide diversity and the large volumes of data that it owns or handles. Although a Telecom Company can benefit in many ways from being involved in such ecosystem and eventually create a new revenue stream $[7,12]$, considerations should be taken into account, regarding the risks that come in front. Since it is a new marketplace, the legal and regulatory environment cannot be considered as stable. Entering a new area always demands careful business approaches. Since a main involvement contains data sharing, trust should also be taken into account, not only technological but 
also ethical which might cause otherwise losing a competitive advantage. Towards that direction, DataPorts project aims at designing a trusted marketplace in order to lower privacy barriers associated with the development of innovative data-intensive applications that consume personal data. The scope is to develop mechanisms that will encourage more and more people (travelers, employees, workers etc.), who so far seem to be reluctant, to share their personal utility and behavioral data. This is very likely to be achieved by keeping data subjects fully informed, including them as actual stakeholders and co-owners of the data archive. The goal is to find the balance between the level of risk the people are willing to take and the benefit they expect as users of a personal data platform; in other words, data privacy versus data utility. The fundamental concern of privacy protection is to prevent confidential data from being leaked. However, in the area of IoT and Big Data, some information about the dataset is desired to be revealed by design. Consequently, the quest is to quantify and control the leakage of sensitive information, so that it remains within a tolerated threshold, while allowing certain types of analytics to be performed. In the following paragraphs indicative techniques to fulfill the privacy requirements are presented:

\section{Privacy and Compliance by Design}

In fact, legislation and privacy norms are becoming increasingly strict, but IT solutions for addressing these issues are lacking. Part of the research in DataPorts project will be to provide solutions for logging and auditing access to sensitive data, modeling, managing and enforcing privacy and consent policies, as well as providing the ability to anonymize sensitive data. With such a solution in place, trust becomes a differentiator while auditing and compliance overhead is decreased for both the data processor and controller. As a result, the business challenge addressed is twofold; (i) the need to prove compliance to privacy and security laws, directives and norms in a more automated and systematic manner with less overhead and (ii) the desire to gain end user trust, encouraging the sharing of personal data and improving the quality of the data shared. The approach aims at providing tools and libraries for privacy and compliance by design and also offering such kind of solutions for existing applications without requiring changes to them. The goal is to make privacy and compliance part of the IT infrastructure and to ensure close coupling of all data with relevant consent and policies.

\section{Privacy Through Data Fuzzification}

In many occasions, people want to share not their actual data but (slightly) different one, trying to balance privacy and accuracy [8]. For example, residents do not wish to expose their exact location, for a variety of purposes, including privacy considerations and risk data leakage that could aid e.g. criminals to understand the occupancy pattern of their house. In the case of utility and behavioral data, the fuzzification could be multidimensional, in terms of space, time and aggregation of the data that are produced from all the smart devices. 


\section{Privacy-Enhanced Decentralized Blockchain-Based Storage System}

The initial idea of the blockchain framework was a permissionless distributed database based on the Bitcoin Peer-2-Peer open-source protocol [6], maintaining a growing list of data records hardened against tampering and revision, even by its own operators. This network model protects users from most prevalent frauds like chargebacks or unwanted charges. DataPorts project aims at moving one step forward in the context of a data marketplace, strengthening the integrity of the framework in terms of privacy. In a decentralized blockchain architecture as such, a full copy of the blockchain contains - at any time - all records of every transaction ever completed in the network and in addition every block contains a hash of the previous block. This enables the blocks to be traced back even to the first one, known as "the genesis block", which makes computationally prohibitively difficult and impractical to modify a block once it is created, especially as the chain of subsequent blocks gets generated [4]. This will protect the privacy-restrictions defined by data subjects, against any possible alternation attempts.

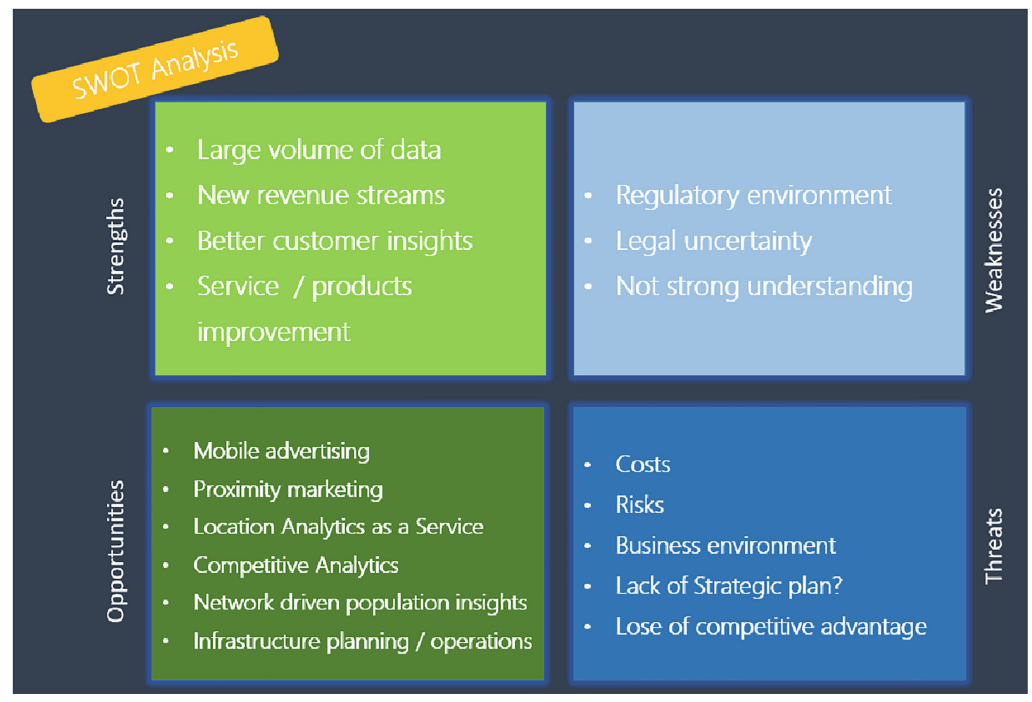

Fig. 4. Challenges \& opportunities for telecom industry - a SWOT analysis

The data that is owned along with their process mechanisms, require Intellectual Property protection, especially within a regulatory complexity. Moreover, the development of data agreements and privacy concerns, as well as, a different than it is used so far, pricing model should be taken under consideration. Risks and potential harms of sharing corporate data, as well as, collecting inaccurate, old or "dirty" data might affect data quality. Since to most of the actions concerning data, the GDPR should be applied, collecting unauthorized 
data or intrusive collection from individuals and organizations could be a complex process. Therefore, improper or unauthorized access to shared data could cause conflicting legal jurisdictions and different security levels and in some cases loss of regulatory licenses, standards and certifications, reputational and industrial damages, as well as, drop in share price and/or increase in cost of capital. Moreover, incomplete or non-representative sampling, or insufficient, outdated or incompatible data sets can be disastrous, especially in the case where there are many recipients of this new data-sharing/trading platform.

Although someone might say that from all the above described parameters, the risk for a Telecom Provider is forbidding enough to enter this new data sharing/trading market, there is equally amount of opportunities to enter these Open data marketplaces $[12,19]$.

\begin{tabular}{lll}
\hline & Business model opportunity & Example \\
\hline 1 & $\begin{array}{l}\text { Product innovators enhance their products and services } \\
\text { with data }\end{array}$ & $\begin{array}{l}\text { Incorporating smart lighting networks in ports through } \\
\text { using data from motion sensors }\end{array}$ \\
\hline 2 & $\begin{array}{l}\text { Systems Innovators use data to integrate multiple } \\
\text { product types }\end{array}$ & $\begin{array}{l}\text { Creating a transport management system using diverse } \\
\text { sources of data (weather sensors, motion sensors, etc.) } \\
\text { and applying insights to multiple devices like bridges, } \\
\text { quays, etc. }\end{array}$ \\
\hline $3 \quad \begin{array}{l}\text { Data Providers gather and sell raw data without adding } \\
\text { too much value to it }\end{array}$ & $\begin{array}{l}\text { Data services like the Automatic identification System } \\
\text { linked to GPS which offers insights in multiple aspects of } \\
\text { ship movements like origin, destination, cargo, etc. }\end{array}$ \\
\hline 4 & $\begin{array}{l}\text { Data Brokers gather and combine data from multiple } \\
\text { sources, create additional value with analytics and sell } \\
\text { insights }\end{array}$ & $\begin{array}{l}\text { Create market reports using multiple sources like order } \\
\text { books and scrapping forecasts }\end{array}$ \\
\hline 5 & $\begin{array}{l}\text { Value Chain Integrators share data with system- } \\
\text { integrator partners to extend product offerings or } \\
\text { reduce costs }\end{array}$ & $\begin{array}{l}\text { Improve the internal port supply chain with data shared by } \\
\text { the port users }\end{array}$ \\
\hline $6 \quad \begin{array}{l}\text { Delivery Network Collaborators share data to drive } \\
\text { deal making, foster marketplaces and enable advertising }\end{array}$ & $\begin{array}{l}\text { Foster the marketplace and drive deal making to a new } \\
\text { level through novel insight driven business models }\end{array}$ \\
\hline
\end{tabular}

Fig. 5. Business opportunities [3]

As it is described in Figs. 4 and 5, it can be an opportunity for new business and increase in customer/subscribers base. In addition, can create an increased availability of vast and heterogeneous data ecosystems for AI and innovative data-driven business models, as well as, a way to tap into 'safe' personal data. Opportunities can also exist for the Telecom subscribers as well, by obtaining control over personal data. It is considered that the well-being and the quality of life benefits from personal data sharing in key sectors. Moreover, opportunities exist by accessing personalized and cross-sectoral B2C services and increasing the potential of personal data monetization. The entry of a Telecom data owner in this emerging data-driven market, creates opportunities for third parties as well. For academia, by increasing the socio-economic impact of research data across domains and borders, it creates an open innovation access through data marketplaces. Last but not least opportunities can be created in general for Government and Public Bodies thus improving the local economy. These bodies could include, among others, the Municipalities and the Regions that host 
the Ports. Common use of data through platforms can lead to improved governmental services, especially AI-enhanced digital services. These local and regional opportunities can also lead to an integrated real-time European analytics system exposing annual statistics of the ports ecosystem.

\section{Conclusion and Future Work}

A Telecom Provider can benefit from entering such rapidly evolving data market. As in every case, there are considerations and risks that should be taken into account. These parameters will be investigated during the Pilots' execution within DataPorts H2020 Project, where various data sets will be provided and used for cognitive applications. During the Piloting Phase, the value of the data and their governance methods will be thoroughly investigated.

\section{References}

1. Agarwal, V., Mittal, S., Mukherjea, S., Dalal, P.: Exploiting rich telecom data for increased monetization of telecom application stores. In: 2012 IEEE 13th International Conference on Mobile Data Management. IEEE, July 2012. https://doi.org/ $10.1109 / \mathrm{mdm} .2012 .28$

2. Banerjee, A.: Big data \& advanced analytics in telecom: a multi-billion-dollar revenue opportunity. Heavy Reading, pp. 1-24 (2013)

3. Berns, S., Dickson, R., Vonck, I., Dragt, J.: Smart Ports (2017). https://www2. deloitte.com/content/dam/Deloitte/nl/Documents/energy-resources/deloitte-nler-port-services-smart-ports.pdf. Accessed 27 Feb 2020

4. Bheemaiah, K.: Why business schools need to teach about the blockchain. SSRN Electron. J. (2015). https://doi.org/10.2139/ssrn.2596465

5. BigDataStack H2020 Project. https://www.bigdatastack.eu. Accessed 27 Feb 2020

6. Bitcoin. https://bitcoin.org/en/. Accessed 27 Feb 2020

7. Bonneau, V.: Data monetisation: opportunities beyond OTT: finance, retail, telecom and connected objects. Commun. Strat. (97), 123 (2015)

8. COSMOS EU Project: End-to-end security and privacy: design and open specification (Final). https://cordis.europa.eu/project/id/609043/reporting. Accessed 27 Feb 2020

9. Danaos Shipping Co., Ltd. https://web2.danaos.gr/research/eu-projects/. Accessed 27 Feb 2020

10. DataPorts H2020 Project. http://dataports-project.eu. Accessed 27 Feb 2020

11. Drougkas, A., Sarri, A., Kyranoudi, P., Zisi, A.: Port cybersecurity - good practices for cybersecurity in the maritime sector, November 2019. https://www.enisa. europa.eu/publications/port-cybersecurity-good-practices-for-cybersecurity-inthe-maritime-sector. Accessed 27 Feb 2020

12. Ducruet, C. (ed.): Advances in Shipping Data Analysis and Modeling. Routledge, London (2017). https://doi.org/10.4324/9781315271446

13. European Sea Ports Organisation. https://www.espo.be/. Accessed 27 Feb 2020

14. Hyperledger Fabric. https://www.hyperledger.org/projects/fabric. Accessed 27 Feb 2020

15. International Association of Ports and Harbors. https://www.iaphworldports.org. Accessed 27 Feb 2020 
16. Jovic, M., Kavran, N., Aksentijevic, S., Tijan, E.: The transition of croatian seaports into smart ports. In: 2019 42nd International Convention on Information and Communication Technology, Electronics and Microelectronics (MIPRO). IEEE, May 2019. https://doi.org/10.23919/mipro.2019.8757111

17. Kim, J., Son, J., Yoon, K.: An implementation of integrated interfaces for telecom systems and TMS in vessels. Int. J. Eng. Technol. 10(2), 195-199 (2018). https:// doi.org/10.7763/ijet.2018.v10.1058

18. Lytra, I., Vidal, M.E., Orlandi, F., Attard, J.: A big data architecture for managing oceans of data and maritime applications. In: 2017 International Conference on Engineering, Technology and Innovation (ICE/ITMC). IEEE, June 2017. https:// doi.org/10.1109/ice.2017.8280019

19. Miele, S., Shockley, R.: Analytics: the real-world use of big data. Retrieved from IBM Institute for Business Value, Said Business School (2013)

20. Molavi, A., Lim, G.J., Race, B.: A framework for building a smart port and smart port index. Int. J. Sustain. Transp., 1-13 (2019). https://doi.org/10.1080/ 15568318.2019.1610919

21. Moulos, V., et al.: A robust information life cycle management framework for securing and governing critical infrastructure systems. Inventions 3(4), 71 (2018). https://doi.org/10.3390/inventions3040071

22. OpenAPI Specification. https://github.com/OAI/OpenAPI-Specification/blob/ master/versions/3.0.3.md. Accessed 04 Mar 2020

23. OpenJS Foundation, Node-RED tool. https://nodered.org/. Accessed 04 Mar 2020

24. PortForward H2020 Project. https://www.portforward-project.eu/. Accessed 27 Feb 2020

25. SmartShip H2020 Project. https://www.smartship2020.eu/. Accessed 27 Feb 2020

26. The JSON Data Interchange Syntax, 2nd edn., December 2017. http://www.ecmainternational.org/publications/files/ECMA-ST/ECMA-404.pdf

27. The Marketplace of the European Innovation Partnership on Smart Cities and Communities. https://eu-smartcities.eu/. Accessed 27 Feb 2020

28. The Worldwide Network of Port Cities. http://www.aivp.org/en/. Accessed 27 Feb 2020

29. Volman, Y.: Report on open data, July 2019. https://ec.europa.eu/digital-singlemarket/en/open-data. Accessed $27 \mathrm{Feb} 2020$

30. Yang, Y., Zhong, M., Yao, H., Yu, F., Fu, X., Postolache, O.: Internet of things for smart ports: technologies and challenges. IEEE Instrum. Meas. Mag. 21(1), 34-43 (2018). https://doi.org/10.1109/mim.2018.8278808 